\title{
NT-proBNP: a useful marker of cardiovascular function in patients on chronic haemodialysis treatment
}

\author{
Gioacchino Li Cavoli', Onofrio Schillaci', Tancredi Vincenzo Li Cavoli ${ }^{1}$, Ugo Rotolo ${ }^{1}$ \\ ${ }^{1}$ Nephrology-Dialysis Department, Civico and Di Cristina Hospital, Palermo, Italy
}

\section{Dear Editor,}

We read with interest the article Relationship between renalase and N-terminal pro-B-type Natriuretic Peptide (NT pro-BNP) in haemodialysis patients [1] and are in agreement with the authors regarding the relevant association between this biomarker and increased cardiovascular diseases (heart failure, stable coronary artery disease and acute coronary syndrome) in haemodialysis (HD) patients. We report our experience on this topic.

For 2 years we studied 47 patients ( 28 males and 19 females) on chronic HD treatment in Palermo-Italy. The mean age and the dialysis procedure were the same as the patients of the province of Lublin. We analyzed the correlation between NT pro-BNP and cardiovascular disease. NT-proBNP was measured by an electrochemiluminescence immunoassay (Elecsys 2010 Roche diagnostic). All patients were studied by echocardiography; we detected left ventricular hypertrophy in $72.4 \%$ of these subjects. During the observation period, 18 patients died. In the subjects, the mean value of NT-
proBNP was $23,931 \mathrm{pg} / \mathrm{ml}$, but in the 13 patients who died from cardiovascular causes, the mean value was considerably higher $(58,638 \mathrm{pg} / \mathrm{ml})$. Analyzing all subjects (alive at the end and died during the study) the overall mean value of NTproBNP was $7,643 \mathrm{pg} / \mathrm{ml}$ and increased to $16,952 \mathrm{pg} / \mathrm{ml}$ at the end of the study. We consider this trend to be the result of multiple elements harmful to the cardiovascular system, and not only for impaired renal clearance.

In conclusion, we think that NT-proBNP is a useful marker of cardiovascular function in HD patients and, as stated by the authors, it should be a part of the routine for cardiovascular risk assessment in these subjects.

\section{REFERENCES}

1. Dziedzic M, Petkowicz B, Bednarek-Skublewska A, Solski J, Buczaj A, Choina P. Relationship between renalase and N-terminal pro-B-type natriuretic peptide (NT pro-BNP) in haemodialysis patients. Ann Agric Environ Med. 2014; 21(1): 132-135. 